\title{
The adaptive brain in mental health: overcoming inherited risk factors
}

\author{
Paul R. Albert, PhD
}

A primary function of the brain is to drive adaptation of the organism to its environment: learning, memory, attachment, fear, aggression, etc., are all manifestations of how the brain directs adaptation to the environment. One touch of a flame in youth is sufficient to engrain a learned behaviour that lasts a lifetime despite the memory of the specific event having long dissipated. To mediate these behaviours, the brain itself must adapt its transcriptional, structural and neurotransmission functions. This type of imprinted memory has been modelled in young Caenorhabditis elegans, where aversive memory is retained throughout the lifetime, while it is forgotten when adults are exposed. ${ }^{1}$ This persistent memory retention requires recruitment of new neurons that alone do not mediate memory retrieval, but that must be activated to permit memory retrieval via other neuronal circuits. Similarly, shocking mice in a given context generates a fear memory by recruiting a sparse subset of hippocampal neurons that can be reactivated to recruit the memory of the context. When these neurons are optogenetically inactivated, the fear memory is extinguished; when activated in a different context, this elicits an inappropriate fear response. ${ }^{2,3}$ Conversely, activating hippocampal cells that respond to a positive memory can reverse stress-induced depression-like behaviours. ${ }^{4}$ Thus, one mechanism of brain adaptation involves cellular adaptation, with recruitment of new sets of neurons.

Despite its potential for adaptation, the greatest risk factor for mental illness remains family history, ${ }^{5}$ implicating genes, environment or both, and suggesting a limited capacity of the brain to adapt to these early and lifelong risk factors. The hypothesis that genetic makeup modifies the trajectory of adaptive behaviours underlies current efforts to identify specific genetic changes associated with mental illness. For example, genes associated with schizophrenia, such as NRG1, DISC1, or DTNBP1, undergo positive selection, suggesting that these genes drive a behavioural phenotype that may confer an evo- lutionary advantage in certain environments. ${ }^{6}$ The question is whether potentially harmful inherited traits provoke homeostatic compensatory responses and whether these responses can be augmented through early interventions.

\section{Brain adaptation to inherited genetic and nongenetic risk in mice}

Studies in mouse models indicate that the brain can adapt to major genetic alterations, such as gene deletions or copy number variations, resulting in "normal" behavioural responses. Focusing on the serotonin (5-HT) system as an example, gene deletion of tryptophan hydroxylase2 (TPH2), the critical enzyme for synthesis of 5-HT in the brain, results in increased aggression and mildly reduced anxiety. ${ }^{7}$ Despite a greater than $90 \%$ loss of brain 5 -HT levels, the $\mathrm{TPH} 2^{-/-}$mice show normal development and firing properties of $5-\mathrm{HT}$ neurons, but show altered 5-HT innervation, ${ }^{8}$ have compensatory upregulation of postsynaptic 5-HT1A and 5-HT1B receptors $^{9,10}$ and reduced 5-HT1A autoreceptor binding, ${ }^{11}$ all of which would amplify the 5-HT system. A similar phenotype is observed in mice with a knock-in of human TPH2 (R439H) mutant resulting in $60 \%-80 \%$ loss of $5-\mathrm{HT}$, with reduced 5-HT1A autoreceptor function with no change in receptor levels. ${ }^{12}$ Mouse genetic models with progressive reduction in $\mathrm{TPH} 2$ activity resulted in no change in brain 5-HT levels or behaviour due to compensation via reductions in 5-HT metabolism and 5-HT1A autoreceptor function. ${ }^{13}$ These studies indicate that molecular adaptation of genes in the 5-HT system compensates for genetic impairments to moderate the behavioural phenotype. This molecular adaptation may involve compensatory gene expression changes that are selected for and engrained by fitness to the environment in the case of these animal models a low-stress, noncompetitive environment.

Correspondence to: P.R. Albert, Ottawa Hospital Research Institute, UOttawa Brain and Mind Research Institute, 451 Smyth Rd, Ottawa ON K1H 8M5; palbert@uottawa.ca

DOI: 10.1503/jpn.160225 
In addition to genetic transmission of traits, nongenetic mechanisms are being uncovered that highlight the complexity of mental illness. For example, transgenerational transmission of 5-HT1A receptor genotype (HTR1A) in prenatal mice has been shown to strongly influence the adult anxiety phenotype. ${ }^{14}$ Embryonic implantation of wild-type HTR1A embryos in $H T R 1 A^{-/-}$mothers transmitted the anxiety phenotype to the first- and second-, but not the third-generation offspring, ${ }^{15}$ suggesting transgenerational adaptation of the behavioural phenotype. This nongenetic maternal transmission of anxiety to wild-type mice appears to involve altered immune function in the offspring, resulting in reduced ventral hippocampal function, associated with altered DNA methylation and expression of lipid and synaptic signalling genes. ${ }^{15,16}$ In humans, the C(-1019)G HTR1A promoter polymorphism associated with depression and suicide prevents regulation by Deaf1, altering brain-wide 5-HT1A receptor expression. ${ }^{17,18}$ In Deaf1 knockout mice, despite similar alterations in 5-HT1A expression, adaptations in the signalling of 5-HT1A receptors across generations led to the development of a mild behavioural phenotype. ${ }^{19}$ Thus, transgenerational molecular adaptation involving changes in gene expression appears to reduce the impact of genetic and nongenetic changes in the HTR1A gene with succeeding generations. By contrast, a mouse model of unpredictable maternal stress and separation from offspring leads to transgenerational transmission across 3 generations of a behavioural phenotype, including impaired social interaction and cognition and increased stress resilience, with persistent changes in pre- and postsynaptic 5-HT1A receptor activity and circuitry. ${ }^{20,21}$ Thus, maternal stress and separation appear to reprogram molecular adaptation to a different affective state that persists for generations.

\section{Enhancing adaptation to inherited risk for depression in humans}

Inter- and transgenerational transmission of depression in humans has been well documented. Children of depressed mothers have higher rates of depression, and children with both depressed mothers and grandmothers had the highest rates compared with children with nondepressed mothers. ${ }^{5}$ Successful treatment of mothers' depression reduces depression in their children, while relapse worsens depression..$^{22,23}$ Interestingly, a 5-HT risk allele for depression (5-HTTLPR s-allele) was 4-fold over-represented in children of mothers with depression, suggesting that genetic risk factors increase susceptibility to maternally transmitted depression. ${ }^{24}$ Similarly, healthy young men with multigenerational history of depression were more susceptible to reducing 5-HT using acute tryptophan depletion (ATD) than those without this family history. ${ }^{25}$ Patients in remission from depression remain vulnerable to ATD, particularly those treated with 5-HT-directed antidepressants. ${ }^{26}$ Thus the ability of the human brain to adapt to inherited risk is incomplete, and the risk of depression persists even after recovery.

How can beneficial brain adaptation to stabilize behaviour be enhanced? One strategy uses pharmacological or other treatments of depression in the mothers, which can enhance the interaction with the child and improve the child's behavioural symptoms. ${ }^{27,28}$ Early treatment of children whose symptoms do not improve despite the mother's remission is another potential strategy. ${ }^{27}$ However, pharmacological approaches to early life treatment in mothers or children may elicit long-term adverse effects. In animal studies, early postnatal ( 44 to p21, equivalent to third trimester [7-9 mo of pregnancy] to $2 \mathrm{yr}$ postnatal in humans ${ }^{29}$ ) treatment with selective serotonin reuptake inhibitors (SSRIs) results in a strong and persistent anxiety- and depression-like phenotype, ${ }^{30}$ and SSRI treatment of children during an analogous developmental window could be detrimental. In animal studies, the effects of treatment of mothers during gestation may also result in anxiety or depression in the offspring ${ }^{31}$ or may lack efficacy. ${ }^{32}$ Treatment of breast-feeding women during the early postpartum period may result in $20 \%$ antidepressant transfer through the milk, but is considered a relatively safe option. ${ }^{33,34}$ However, there is some evidence of behavioural or psychomotor impairment in human infants following prenatal exposure to SSRIs, but further study is needed. ${ }^{35}$ Exposure of children to maternal depression from age $2-5$ years appears to be a critical period for transmission of behavioural disturbances, ${ }^{36}$ hence this period may be the most important and perhaps safer to target for treatment. It remains unclear whether antidepressant treatment at these early developmental stages results in cellular, molecular or both forms of brain adaptation.

An attractive alternative to pharmacological treatment of children is nonpharmacological treatment to enhance the child's developmental environment. The finding that children of depressed mothers are at high risk for depression, and that this risk is diminished with successful treatment of the mother suggests that, while genetic transmission of risk may play a role, a key determinant of the child's mental health is a nurturing maternal environment. Thus, a major approach to enhancing brain adaptation to genetic risk would be to effectively treat the mother as early as possible (with antidepressants and/or psychotherapy) and to enhance the mother-child relationship during the critical developmental period between ages $2-5$ years. The latter nonpharmacological approach could include family support and education to ensure that the child receives quality parenting while the mother is depressed, careful monitoring of the child for signs of anxiety or depression, and psychotherapy should such signs emerge - all of which may enhance brain adaptation.

\section{Conclusion}

There is evidence for molecular brain adaptations to inherited vulnerability to anxiety and depression that can sometimes occur over generations. These processes can be accelerated by interventions that improve the environment, or that directly target brain plasticity. Future studies of the mechanisms underlying early intervention strategies to enhance brain adaptation may provide new approaches to treat vulnerable populations. 
Affiliation: From the Ottawa Hospital Research Institute, UOttawa Brain and Mind Research Institute, Ottawa, Ont., Canada.

Competing interests: None declared.

\section{References}

1. Jin X, Pokala N, Bargmann CI. Distinct circuits for the formation and retrieval of an imprinted olfactory memory. Cell 2016; 164:632-43.

2. Denny CA, Kheirbek MA, Alba EL, et al. Hippocampal memory traces are differentially modulated by experience, time, and adult neurogenesis. Neuron 2014;83:189-201.

3. Liu X, Ramirez S, Pang PT, et al. Optogenetic stimulation of a hippocampal engram activates fear memory recall. Nature 2012; 484:381-5.

4. Ramirez S, Liu X, MacDonald CJ, et al. Activating positive memory engrams suppresses depression-like behaviour. Nature 2015; 522:335-9.

5. Weissman MM, Berry OO, Warner V, et al. A 30-year study of 3 generations at high risk and low risk for depression. JAMA Psychiatry 2016;73:970-7.

6. Crespi B, Summers K, Dorus S. Adaptive evolution of genes underlying schizophrenia. Proc Biol Sci 2007;274:2801-10.

7. Mosienko V, Bert B, Beis D, et al. Exaggerated aggression and decreased anxiety in mice deficient in brain serotonin. Transl Psychiatry 2012;2:e122.

8. Migliarini S, Pacini G, Pelosi B, et al. Lack of brain serotonin affects postnatal development and serotonergic neuronal circuitry formation. Mol Psychiatry 2013;18:1106-18.

9. Gutknecht L, Araragi N, Merker S, et al. Impacts of brain serotonin deficiency following Tph2 inactivation on development and raphe neuron serotonergic specification. PLoS One 2012;7:e43157.

10. Montalbano A, Waider J, Barbieri M, et al. Cellular resilience: 5-HT neurons in Tph2(-/-) mice retain normal firing behavior despite the lack of brain 5-HT. Eur Neuropsychopharmacol 2015;25:2022-35.

11. Kim JY, Kim A, Zhao ZQ, et al. Postnatal maintenance of the 5 -Ht1a-Pet1 autoregulatory loop by serotonin in the raphe nuclei of the brainstem. Mol Brain 2014;7:48.

12. Jacobsen JP, Siesser WB, Sachs BD, et al. Deficient serotonin neurotransmission and depression-like serotonin biomarker alterations in tryptophan hydroxylase 2 (Tph2) loss-of-function mice. Mol Psychiatry 2012;17:694-704.

13. Mosienko V, Matthes S, Hirth N, et al. Adaptive changes in serotonin metabolism preserve normal behavior in mice with reduced TPH2 activity. Neuropharmacology 2014;85:73-80.

14. Gleason G, Liu B, Bruening S, et al. The serotonin1A receptor gene as a genetic and prenatal maternal environmental factor in anxiety. Proc Natl Acad Sci U S A 2010;107:7592-7.

15. Mitchell E, Klein SL, Argyropoulos KV, et al. Behavioural traits propagate across generations via segregated iterative-somatic and gametic epigenetic mechanisms. Nat Commun 2016;7:11492.

16. Oh JE, Chambwe N, Klein S, et al. Differential gene body methylation and reduced expression of cell adhesion and neurotransmitter receptor genes in adverse maternal environment. Transl Psychiatry 2013;3:e218

17. Albert PR, Lemonde S. 5-HT1A receptors, gene repression, and depression: guilt by association. Neuroscientist 2004;10:575-93

18. Donaldson ZR, Le Francois B, Santos TL, et al. The functional serotonin 1a receptor promoter polymorphism, rs6295, is associated with psychiatric illness and differences in transcription. Transl Psychiatry 2016;6:e746.

19. Luckhart C, Philippe TJ, Le Francois B, et al. Sex-dependent adaptive changes in serotonin-1A autoreceptor function and anxiety in Deaf1-deficient mice. Mol Brain 2016;9:77.

20. Franklin TB, Linder N, Russig H, et al. Influence of early stress on social abilities and serotonergic functions across generations in mice. PLoS One 2011;6:e21842.

21. Razoux F, Russig H, Mueggler T, et al. Transgenerational disruption of functional 5-HT1AR-induced connectivity in the adult mouse brain by traumatic stress in early life. Mol Psychiatry 2016; doi: 10.1038 .

22. Weissman MM, Olfson M. Translating intergenerational research on depression into clinical practice. JAMA 2009;302:2695-6.

23. Weissman MM, Wickramaratne P, Pilowsky DJ, et al. The effects on children of depressed mothers' remission and relapse over 9 months. Psychol Med 2014;44:2811-24.

24. Talati A, Guffanti G, Odgerel Z, et al. Genetic variants within the serotonin transporter associated with familial risk for major depression. Psychiatry Res 2015;228:170-3.

25. Benkelfat C, Ellenbogen MA, Dean P, et al. Mood-lowering effect of tryptophan depletion. Enhanced susceptibility in young men at genetic risk for major affective disorders. Arch Gen Psychiatry 1994;51:687-97.

26. Ruhé HG, Mason NS, Schene AH. Mood is indirectly related to serotonin, norepinephrine and dopamine levels in humans: a meta-analysis of monoamine depletion studies. Mol Psychiatry 2007;12:331-59.

27. Batten LA, Hernandez M, Pilowsky DJ, et al. Children of treatment-seeking depressed mothers: a comparison with the sequenced treatment alternatives to relieve depression $\left(S T A R^{*} D\right)$ child study. J Am Acad Child Adolesc Psychiatry 2012;51:1185-96.

28. Weissman MM, Wickramaratne P, Pilowsky DJ, et al. Treatment of maternal depression in a medication clinical trial and its effect on children. Am J Psychiatry 2015;172:450-9.

29. Semple BD, Blomgren K, Gimlin K, et al. Brain development in rodents and humans: identifying benchmarks of maturation and vulnerability to injury across species. Prog Neurobiol 2013;106-107:1-16.

30. Ansorge MS, Zhou M, Lira A, et al. Early-life blockade of the 5-HT transporter alters emotional behavior in adult mice. Science 2004; 306:879-81.

31. Olivier JD, Valles A, van Heesch F, et al. Fluoxetine administration to pregnant rats increases anxiety-related behavior in the offspring. Psychopharmacology (Berl) 2011;217:419-32.

32. Zohar I, Shoham S, Weinstock M. Perinatal citalopram does not prevent the effect of prenatal stress on anxiety, depressive-like behaviour and serotonergic transmission in adult rat offspring. Eur $J$ Neurosci 2016;43:590-600.

33. Weissman AM, Levy BT, Hartz AJ, et al. Pooled analysis of antidepressant levels in lactating mothers, breast milk, and nursing infants. Am J Psychiatry 2004;161:1066-78.

34. Lanza di Scalea T, Wisner KL. Antidepressant medication use during breastfeeding. Clin Obstet Gynecol 2009;52:483-97.

35. Oberlander TF, Gingrich JA, Ansorge MS. Sustained neurobehavioral effects of exposure to SSRI antidepressants during development: molecular to clinical evidence. Clin Pharmacol Ther 2009; 86:672-7.

36. Naicker K, Wickham M, Colman I. Timing of first exposure to maternal depression and adolescent emotional disorder in a national Canadian cohort. PLoS One 2012;7:e33422. 